\title{
THE MULTIPLE GAMMA FUNCTION AND ITS $q$-ANALOGUE
}

\author{
KIMIO UENO and MICHITOMO NISHIZAWA \\ Department of Mathematics \\ School of Science and Engineering, Waseda University \\ 3-4-1 Okubo, Shinjuku-ku, Tokyo 169, Japan \\ E-mail:uenoki@cfi.waseda.ac.jp and694m5035@cfi.waseda.ac.jp
}

\begin{abstract}
We give an asymptotic expansion (the higher Stirling formula) and an infinite product representation (the Weierstrass product formula) of the Vignéras multiple gamma function by considering the classical limit of the multiple $q$-gamma function.

1. Introduction. The multiple gamma function was introduced by Barnes. It is defined to be an infinite product regularized by the multiple Hurwitz zeta-functions [2], [3], [4], [5]. After his discovery, many mathematicians have studied this function: Hardy [7], [8] studied this function from his viewpoint of the theory of elliptic functions, and Shintani [20], [21] applied it to the study on the Kronecker limit formula for zeta-functions attached to algebraic fields.

In the end of 70's, Vignéras [25] redefined a multiple gamma function to be a function satisfying the generalized Bohr-Morellup theorem. Furthermore, Vignéras [25], Voros [26], and Kurokawa [12], [13], [14], [15] showed that it plays an essential role to express the Selberg zeta-function and the determinant of Laplacians.

As we can see from these studies, the multiple gamma function is a fundamental function for the analytic number theory: See also [16], [17]. However we do not think that the theory of the multiple gamma functions has been fully explored.

On the other hand, the second author of this article introduced a $q$-analogue of the Vignéras multiple gamma functions and showed it to be characterized by a $q$-analogue of the generalized Bohr-Morellup theorem [25].

In this article, we will establish an asymptotic expansion formula (the higher Stirling formula) and an infinite product representation (the Weierstrass product formula) of the Vignéras multiple gamma function by considering the classical limit of the multiple $q$-gamma functions. In order to get these results, we will use the method developed in
\end{abstract}

1991 Mathematics Subject Classification: 33B15, 33D05.

The paper is in final form and no version of it will be published elsewhere. 
[22]. Namely, by making use of the Euler-MacLaurin summation formula, we derive the Euler-MacLaurin expansion of the multiple $q$-gamma function. Taking the classical limit, we lead to the Euler-MacLaurin expansion of the Vignéras multiple gamma function. The higher Stirling formula and the Weierstrass product formula follow from this expansion formula

The details of the proof will be published in the forthcoming paper [23].

2. A survey of the multiple gamma function and the multiple $q$-gamma function

2.1. The gamma function. The following are well-known facts in the classical analysis: The Bohr-Morellup theorem says that the gamma function $\Gamma(z)$ is characterized by the three conditions,

1. $\Gamma(z+1)=z \Gamma(z)$,

2. $\Gamma(1)=1$,

3. $\frac{d^{2}}{d z^{2}} \log \Gamma(z+1) \geq 0$ for $z \geq 0$.

The gamma function is meromorphic on $\mathbf{C}$, and has an infinite product representation

$$
\Gamma(z+1)=e^{-\gamma x} \prod_{n=1}^{\infty}\left\{\left(1+\frac{z}{n}\right)^{-1} e^{\frac{z}{n}}\right\},
$$

where $\gamma$ is the Euler Constant. This is usually called the Weierstrass product formula.

The gamma function has an asymptotic expansion, which is called the Stirling formula,

$$
\begin{aligned}
& \log \Gamma(z+1) \sim\left(z+\frac{1}{2}\right) \log (z+1)-(z+1)-\zeta^{\prime}(0) \\
& +\sum_{r=1}^{\infty} \frac{B_{2 r}}{[2 r]_{2}} \frac{1}{(z+1)^{2 r-1}}
\end{aligned}
$$

as $z \rightarrow \infty$ in the sector $\Delta_{\delta}:=\{z \in \mathbf{C}|| \arg z \mid<\pi-\delta\}(0<\delta<\pi)$, where

$$
\frac{z e^{t z}}{e^{z}-1}=\sum_{n=0}^{\infty} \frac{B_{n}(t)}{n !} z^{n}
$$

$B_{k}=B_{k}(0)$ (the Bernoulli number), $\zeta(s)$ is the Riemann zeta-function, $\zeta^{\prime}(s)=\frac{d}{d s} \zeta(s)$ and $[x]_{r}=x(x-1) \cdots(x-r+1)$. Note that $\zeta^{\prime}(0)=-\log \sqrt{2 \pi}$.

2.2. The Barnes G-function. Barnes [2] introduced the function $G(z)$ which satisfies

1. $G(z+1)=\Gamma(z) G(z)$,

2. $G(1)=1$,

3. $\frac{d^{3}}{d z^{3}} \log G(z+1) \geq 0$ for $z \geq 0$,

and he called this " $G$-function". He proved that the $G$-function has an infinite product representation

$$
G(z+1)=e^{-z \zeta^{\prime}(0)-\frac{z^{2}}{2} \gamma-\frac{z^{2}+z}{2}} \prod_{k=1}^{\infty}\left\{\left(1+\frac{z}{k}\right)^{k} \exp \left(-z+\frac{z^{2}}{2 k}\right)\right\}
$$


and an asymptotic expansion

$$
\log G(z+1) \sim\left(\frac{z^{2}}{2}-\frac{1}{12}\right) \log (z+1)-\frac{3}{4} z^{2}-\frac{z}{2}+\frac{1}{3}+z \zeta^{\prime}(0)-\log A+O\left(\frac{1}{z}\right)
$$

as $z \rightarrow \infty$ in the sector $\Delta_{\delta}$ where $A$ is called the Kinkelin constant. Voros showed this constant can be written with the first derivative of the Riemann zeta-function (cf [26], $[24])$

$$
\log A=-\zeta^{\prime}(-1)+\frac{1}{12}
$$

2.3. The Vignéras multiple gamma function. As a generalization of the gamma function and the $G$-function, Vignéras [25] introduced a hierarchy of functions which satisfy

$$
\begin{aligned}
& \text { 1. } G_{n}(z+1)=G_{n-1}(z) G_{n}(z), \\
& \text { 2. } G_{n}(1)=1, \\
& \text { 3. } \frac{d^{n+1}}{d z^{n+1}} \log G_{n}(z+1) \geq 0 \text { for } z \geq 0, \\
& \text { 4. } G_{0}(z)=z,
\end{aligned}
$$

and she called these functions "the multiple gamma functions". Applying Dufresnoy and Pisot's results [6], she showed that these functions satisfying the above properties are unique and that it has an infinite product representation:

$$
\begin{aligned}
& G_{n}(z+1)=\exp \left[-z E_{n}(1)+\sum_{h=1}^{n-1} \frac{p_{h}(z)}{h !}\left(\psi_{n-1}^{(h)}(0)-E_{n}^{(h)}(1)\right)\right] \\
& \times \prod_{\mathbf{m} \in \mathbf{N}^{n-1} \times \mathbf{N}^{*}}\left[\left(1+\frac{z}{s(\mathbf{m})}\right)^{(-1)^{n}} \exp \left\{\sum_{l=0}^{n-1} \frac{(-1)^{n-l}}{n-l}\left(\frac{z}{s(\mathbf{m})}\right)^{n-l}\right\}\right]
\end{aligned}
$$

where

$$
\begin{aligned}
& E_{n}(z):=\sum_{\mathbf{m} \in \mathbf{N}^{n-1} \times \mathbf{N}^{*}}\left[\left\{\sum_{l=0}^{n-1} \frac{(-1)^{n-l}}{n-l}\left(\frac{z}{s(\mathbf{m})}\right)^{n-l}\right\}+(-1)^{n} \log \left(1+\frac{z}{s(\mathbf{m})}\right)\right], \\
& \psi_{n-1}(z):=\log G_{n-1}(z+1), \\
& \frac{e^{t z}-1}{e^{z}-1}:=1+\sum_{k=0}^{\infty} p_{k}(t) \frac{z^{k}}{k !}, \\
& s(\mathbf{m}):=m_{1}+m_{2}+\cdots+m_{n} \quad \text { for } \quad \mathbf{m}=\left(m_{1}, m_{2}, \cdots m_{n}\right),
\end{aligned}
$$

and $\mathbf{N}^{*}=\mathbf{N}-\{0\}$.

2.4. The q-gamma function. Throughout this article, we suppose $0<q<1$. A $q$ analogue of the gamma function was defined by Jackson [9], [10]:

$$
\Gamma(z+1 ; q)=(1-q)^{-z} \prod_{k=1}^{\infty}\left(\frac{1-q^{z+k}}{1-q^{k}}\right)^{-1} .
$$


Askey [1] pointed out that this function satisfies a $q$-analogues of the Bohr-Morellup theorem. Namely, $\Gamma(z ; q)$ satisfies

1. $\Gamma(z+1 ; q)=[z] \Gamma(z ; q)$,

2. $\Gamma(1 ; q)=1$,

3. $\frac{d^{2}}{d z^{2}} \log \Gamma(z+1 ; q) \geq 0$ for $z \geq 0$,

where $[z]:=\left(1-q^{z}\right) /(1-q)$.

As $q$ tends to $1-0, \Gamma(z ; q)$ converges $\Gamma(z)$ uniformly with respect to $z$. A rigorous proof of this fact was given by Koornwinder [11].

Inspired by Moak's works [18], the authors [22] derived a representation of the $q$ gamma function

$$
\begin{aligned}
\log \Gamma(z: q) & =\left(z-\frac{1}{2}\right) \log \left(\frac{1-q^{z}}{1-q}\right)+\log q \int_{1}^{z} \xi \frac{q^{\xi}}{1-q^{\xi}} d \xi \\
& +C_{1}(q)+\frac{1}{12} \log q+\sum_{k=1}^{\infty} \frac{B_{2 k}}{(2 k) !}\left(\frac{\log q}{q^{z}-1}\right)^{2 k-1} \widetilde{M}_{2 k-1}\left(q^{z}\right) \\
& +R_{2 m}(z ; q)
\end{aligned}
$$

where

$$
\begin{aligned}
& C_{1}(q)=-\frac{1}{12} \log q-\frac{1}{12} \frac{\log q}{q-1}+\int_{0}^{\infty} \frac{\bar{B}_{2}(t)}{2}\left(\frac{\log q}{q^{t+1}-1}\right)^{2} q^{t+1} d t \\
& R_{2 m}(z: q)=\int_{0}^{\infty} \frac{\bar{B}_{2 m}(t)}{(2 m) !}\left(\frac{\log q}{q^{t+z}-1}\right)^{2 m} \widetilde{M}_{2 m}\left(q^{t+z}\right) d z
\end{aligned}
$$

and the polynomial $\widetilde{M}_{n}(x)$ is defined by the recurrence formula

$$
\widetilde{M}_{1}(x)=1, \quad\left(x^{2}-x\right) \frac{d}{d x} \widetilde{M}_{n}(x)+n x \widetilde{M}_{n}(x)=\widetilde{M}_{n+1}(x) .
$$

Each term of the formula (4) converges uniformly as $q \rightarrow 1-0$. So we get another proof of the uniformity of the classical limit of $\log \Gamma(z ; q)$.

2.5. The multiple q-gamma function. Recently, one of the authors [19] construct the function $G_{n}(z ; q)$ which satisfies a $q$-analogue of the generalized Bohr-Morellup theorem

1. $G_{n}(z+1 ; q)=G_{n-1}(z ; q) G_{n}(z ; q)$,

2. $G_{n}(1 ; q)=1$,

3. $\frac{d^{n+1}}{d z^{n+1}} \log G_{n+1}(z+1 ; q) \geq 0$ for $z \geq 0$,

4. $G_{0}(z ; q)=[z]$.

We call it "the multiple $q$-gamma function". It is given by the following infinite product representation:

$$
G_{0}(z+1 ; q):=[z+1]
$$




$$
\begin{aligned}
G_{n}(z+1 ; q) & :=(1-q)^{-\left(\begin{array}{c}
z \\
n
\end{array}\right)} \prod_{k=1}^{\infty}\left\{\left(\frac{1-q^{z+k}}{1-q^{k}}\right)^{\left(\begin{array}{c}
-k \\
n-1
\end{array}\right)}\left(1-q^{k}\right)^{g_{n}(z, k)}\right\} \\
(n \geq 1), &
\end{aligned}
$$

where

$$
g_{n}(z ; u)=\left(\begin{array}{c}
z-u \\
n-1
\end{array}\right)-\left(\begin{array}{c}
-u \\
n-1
\end{array}\right)
$$

In the next section, we derive a representation of the multiple $q$-gamma function like (4) and consider its classical limit. This limit formula gives some important properties of the multiple gamma function.

3. The Euler-MacLaurin expansion. Our main tool is the Euler-MacLaurin summation formula

$$
\begin{aligned}
\sum_{r=M}^{N-1} f(r)=\int_{M}^{N} f(t) d t & +\sum_{k=1}^{n} \frac{B_{k}}{k !}\left\{f^{(k-1)}(N)-f^{(k-1)}(M)\right\} \\
& +(-1)^{n-1} \int_{M}^{N} \frac{\bar{B}_{n}(t)}{n !} f^{(n)}(t) d t
\end{aligned}
$$

where $\bar{B}_{n}(t)=B_{n}(t-[t])$ and $[t]$ denotes the integral part of $t$. We apply this to $\log G_{n}(z+$ $1 ; q)$.

From the infinite product representation (5), we have

$$
\begin{aligned}
\log G_{n}(z+1 ; q) & =-\left(\begin{array}{l}
z \\
n
\end{array}\right) \log (1-q)-\sum_{k=1}^{\infty}\left(\begin{array}{c}
-k \\
n-1
\end{array}\right) \log \left(1-q^{z+k}\right) \\
& +\sum_{j=0}^{n-1} G_{n, j}(z)\left\{\sum_{k=1}^{\infty} k^{j} \log \left(1-q^{k}\right)\right\},
\end{aligned}
$$

where

$$
\left(\begin{array}{c}
z-u \\
n-1
\end{array}\right)=\sum_{j=0}^{n-1} G_{n, j}(z) u^{j} .
$$

By the Euler-MacLaurin summation formula, we obtain

$$
\begin{aligned}
& \sum_{k=1}^{\infty}\left(\begin{array}{c}
-k \\
n-1
\end{array}\right) \log \left(1-q^{z+k}\right) \\
& \quad=\sum_{j=0}^{n-1} \frac{(-1)^{j}{ }_{n-1} S_{j}}{(n-1) !} \sum_{r=0}^{j} \frac{(-1)^{r} j !}{(j-r) !} L_{r+2}(z+1) \\
& \quad+\left.\sum_{r=1}^{m} \frac{B_{r}}{r !}\left\{\left(\frac{d}{d t}\right)^{r-1}\left(\begin{array}{c}
-t \\
n-1
\end{array}\right)\right\}\right|_{t=1} L_{1} \\
& \quad-\sum_{r=1}^{m} \frac{B_{r}}{r !} F_{n, r-1}(z ; q)+R_{n, m}(z ; q),
\end{aligned}
$$


where

$$
\begin{aligned}
& L_{r}(z):=\frac{L i_{r}\left(q^{z}\right)}{\log ^{r-1} q}, \quad L_{1}(z):=-\log \left(1-q^{z}\right), \quad L_{r}:=L_{r}(1), \\
& L i_{r}(z):=\sum_{k=1}^{\infty} \frac{z^{k}}{k^{r}} \quad \text { (Euler's polylogarithm), } \\
& F_{n, r-1}(z ; q):=\left[\frac{d^{r-1}}{d t^{r-1}}\left\{\left(\begin{array}{c}
-t \\
n-1
\end{array}\right) \log \left(\frac{1-q^{z+t}}{1-q^{z+1}}\right)\right\}\right]_{t=1} \\
& R_{n, m}(z ; q):=\frac{(-1)^{m-1}}{m !} \int_{1}^{\infty}\left[\bar{B}_{m}(t)\left\{\frac{d^{m}}{d t^{m}}\left\{\left(\begin{array}{c}
-t \\
n-1
\end{array}\right) \log \left(\frac{1-q^{z+t}}{1-q^{z+1}}\right)\right\}\right\}\right] d t,
\end{aligned}
$$

and ${ }_{n} S_{j}$ is the Stirling number of the first kind defined by

Similarly, we have

$$
\sum_{j=0}^{n} n S_{j} u^{j}=u(u-1)(u-2) \cdots(u-n+1) .
$$

$$
\sum_{k=1}^{\infty} k^{j} \log \left(1-q^{k}\right)=\sum_{r=0}^{j} \frac{(-1)^{r} j !}{(j-r) !} L_{r+2}+\sum_{r=1}^{j} \frac{B_{r}}{r !} \frac{j !}{(j+1-r) !} L_{1}+C_{j}(q),
$$

where

$$
\begin{aligned}
& C_{j}(q):=-\sum_{r=1}^{n+1} \frac{B_{r}}{r !} f_{j+1, r-1}(q) \\
&+\frac{(-1)^{n}}{(n+1) !} \int_{1}^{\infty}\left[\bar{B}_{n+1}(t)\left\{\frac{d^{n+1}}{d t^{n+1}}\left\{t^{j} \log \left(\frac{1-q^{t}}{1-q}\right)\right\}\right\}\right] d t, \\
& f_{j+1, r-1}(q):=\left[\frac{d^{r-1}}{d t^{r-1}}\left\{t^{j} \log \left(\frac{1-q^{t}}{1-q}\right)\right\}\right]_{t=1} .
\end{aligned}
$$

Substituting (7) and (8) to (6), we have

$$
\begin{aligned}
& \log G_{n}(z+1 ; q) \\
& =\left(\text { the terms containing } L_{r}(z) \text { and } L_{r}\right) \\
& \quad+(\text { the term which converges as } q \rightarrow 1) .
\end{aligned}
$$

$L_{r}(z)$ and $L_{r}$ give rise to a divergent part in the expression (9) as $q \rightarrow 1-0$. But, thanks to the identity

$$
\begin{aligned}
L_{l+1}(z) & =\frac{z^{l}}{l !} \log \left(\frac{1-q^{z}}{1-q}\right) \\
& +\sum_{r=0}^{l} \frac{(z-1)^{l-r}}{(l-r) !} L_{r+1}+\sum_{r=1}^{l} \frac{(-1)^{l} z^{l-r}}{(l-r) !} \int_{1}^{z} \frac{\xi^{r}}{r !} \frac{q^{\xi} \log q}{1-q^{\xi}} d \xi,
\end{aligned}
$$

we can show that the first term of $(9)$ vanishes. Therefore, $\log G_{n}(z+1 ; q)$ reads as follows: 
Proposition 1. Suppose $\Re z>-1$ and $m>n$. Then we have

$$
\begin{aligned}
\log G_{n}(z+1 ; q) & =\left\{\left(\begin{array}{c}
z+1 \\
n
\end{array}\right)+\sum_{r=1}^{n} \frac{B_{r}}{r !}\left(-\frac{d}{d z}\right)^{r-1}\left(\begin{array}{c}
z \\
n-1
\end{array}\right)\right\} \log \left(\frac{1-q^{z+1}}{1-q}\right) \\
+ & \sum_{r=1}^{n}\left\{\left(-\frac{d}{d z}\right)^{r-1}\left(\begin{array}{c}
z \\
n-1
\end{array}\right)\right\} \times \int_{1}^{z+1} \frac{\xi^{r}}{r !} \frac{q^{\xi} \log q}{1-q^{\xi}} d \xi \\
+ & \sum_{j=0}^{n-1} G_{n, j}(z) C_{j}(q)+\sum_{r=1}^{m} \frac{B_{r}}{r !} F_{n, r-1}(z ; q)-R_{n, m}(z ; q) .
\end{aligned}
$$

4. Classical limit. Now, let us calculate the classical limit. As $q \rightarrow 1-0$, we see that for $\Re z>-1$,

$$
\begin{aligned}
& \text { (i) } \log \left(\frac{1-q^{z+1}}{1-q}\right) \rightarrow \log (z+1), \\
& \text { (ii) } \int_{1}^{z+1} \frac{\xi^{r}}{r !} \frac{q^{\xi} \log q}{1-q^{\xi}} d \xi \rightarrow-\int_{1}^{\infty} \frac{\xi^{r-1}}{r !} d \xi=-\frac{1}{r ! r}\left\{(z+1)^{r}-1\right\}, \\
& \text { (iii) } F_{n, r-1}(z ; q) \rightarrow F_{n, r-1}(z), \\
& \text { (iv) } R_{n, m}(z ; q) \rightarrow R_{n, m}(z), \\
& \text { (v) } C_{j}(q) \rightarrow C_{j}:=-\left.\sum_{r=1}^{n+1} \frac{B_{r}}{r !}\left(\frac{d}{d t}\right)^{r-1}\left\{t^{j} \log t\right\}\right|_{t=1} ^{(-1)^{n}} \int_{1}^{\infty} \bar{B}_{n+1}(t)\left(\frac{d}{d t}\right)^{n+1}\left\{t^{j} \log t\right\} d t
\end{aligned}
$$

where

$$
\begin{aligned}
& F_{n, r-1}(z):=\left.\left(\frac{d}{d t}\right)^{r-1}\left\{\left(\begin{array}{c}
-t \\
n-1
\end{array}\right) \log \left(\frac{z+t}{z+1}\right)\right\}\right|_{t=1}, \\
& R_{n, m}(z):=\frac{(-1)^{m-1}}{m !} \int_{1}^{\infty} \bar{B}_{m}(t)\left(\frac{d}{d t}\right)^{m}\left\{\left(\begin{array}{c}
-t \\
n-1
\end{array}\right) \log \left(\frac{z+t}{z+1}\right)\right\} d t .
\end{aligned}
$$

Furthermore, from Vitali's convergence theorem, we can show that the convergence is uniform on any compact set in $\{\Re z>-1\}$.

The constant $C_{j}$ is relevant to the Riemann zeta-function. Indeed, we obtain

$$
C_{j}=-\exp \left(\zeta^{\prime}(-j)\right)-\frac{1}{(j+1)^{2}}
$$

by applying the Euler-MacLaurin summation formula. 
Thus we have proved the existence of the classical limit of $G_{n}(z+1 ; q)$, and put it to be

$$
\tilde{G}_{n}(z+1):=\lim _{q \rightarrow 1-0} G_{n}(z+1 ; q) .
$$

The uniformity of the convergence ensures that $\tilde{G}_{n}(z+1)$ satisfies

1. $\tilde{G}_{n}(z+1)=\tilde{G}_{n-1}(z) \tilde{G}_{n}(z)$,

2. $\left(\frac{d}{d z}\right)^{n+1} \log \tilde{G}_{n}(z+1) \geq 0$ for $z \geq 0$,

3. $\tilde{G}_{n}(1)=1$,

4. $\tilde{G}_{0}(z+1)=z+1$.

From the uniqueness of the function satisfying these conditions, we have

$$
\tilde{G}_{n}(z+1)=G_{n}(z+1) \text { for } \Re z>-1 .
$$

Namely, as $q \rightarrow 1-0$,

$$
G_{n}(z+1 ; q) \rightarrow G_{n}(z+1) \quad \text { in } \quad\{\Re z>-1\} .
$$

Using the functional equation $G_{n}(z+1)=G_{n-1}(z) G_{n}(z)$, we can show the following theorem.

THEOREM 2. As $q \rightarrow 1-0, G_{n}(z+1 ; q)$ converges $G_{n}(z+1)$ uniformly on any compact set in the domain $\mathbf{C} \backslash \mathbf{Z}_{<0}$, and

$$
\begin{aligned}
\log & G_{n}(z+1) \\
= & \left\{\left(\begin{array}{c}
z+1 \\
n
\end{array}\right)+\sum_{r=1}^{n} \frac{B_{r}}{r !}\left(-\frac{d}{d z}\right)^{r-1}\left(\begin{array}{c}
z \\
n-1
\end{array}\right)\right\} \log (z+1) \\
& -\sum_{r=1}^{n}\left\{\left(-\frac{d}{d z}\right)^{r-1}\left(\begin{array}{c}
z \\
n-1
\end{array}\right)\right\} \times \frac{1}{r ! r}\left\{(z+1)^{r}-1\right\} \\
& -\sum_{j=0}^{n-1} G_{n, j}(z)\left\{\zeta^{\prime}(-j)+\frac{1}{(j+1)^{2}}\right\}+\sum_{r=1}^{\infty} \frac{B_{r}}{r !} F_{n, r-1}(z) \\
- & R_{n, m}(z) .
\end{aligned}
$$

5. The higher Stirling formula. As $|z| \rightarrow \infty$ in $\Delta_{\delta}$, we can see that

$$
\left|R_{n, m}(z)\right|=O\left(z^{n-m+1}\right), \quad\left|F_{m, r-1}(z)\right|=O\left(z^{-r+n}\right) .
$$

Thus, we have proved the higher Stirling formula:

THEOREM 3. As $|z| \rightarrow \infty$ in the sector $\Delta_{\delta}$, we obtain

$$
\begin{aligned}
& \log G_{n}(z+1) \\
& \quad \sim\left\{\left(\begin{array}{c}
z+1 \\
n
\end{array}\right)+\sum_{r=1}^{n} \frac{B_{r}}{r !}\left(-\frac{d}{d z}\right)^{r-1}\left(\begin{array}{c}
z \\
n-1
\end{array}\right)\right\} \log (z+1)
\end{aligned}
$$




$$
\begin{aligned}
& -\sum_{r=1}^{n}\left\{\left(-\frac{d}{d z}\right)^{r-1}\left(\begin{array}{c}
z \\
n-1
\end{array}\right)\right\} \times \frac{1}{r ! r}\left\{(z+1)^{r}-1\right\} \\
& -\sum_{j=0}^{n-1} G_{n, j}(z)\left\{\zeta^{\prime}(-j)+\frac{1}{(j+1)^{2}}\right\}+\sum_{r=1}^{\infty} \frac{B_{2 r}}{(2 r) !} F_{n, 2 r-1}(z) .
\end{aligned}
$$

Examples of the higher Stirling formula. Let us show some examples. In the case that $n=1$, this formula coincides with the Stirling formula. In each case that $n=2$, it coincides with (2). Namely, we have

$$
\begin{aligned}
& \log G_{2}(z+1) \\
& \sim\left(\frac{z^{2}}{2}-\frac{1}{12}\right) \log (z+1)-\frac{3}{4} z^{2}-\frac{z}{2}+\frac{1}{4}-z \zeta^{\prime}(0)+\zeta^{\prime}(-1) \\
& -\frac{1}{12} \frac{1}{z+1}+\sum_{r=2}^{\infty} \frac{B_{2 r}}{[2 r]_{3}} \frac{1}{(z+1)^{2 r-1}}(z-2 r+1) .
\end{aligned}
$$

In the case that $n=3$ and $n=4$, we obtain

$$
\begin{aligned}
\log G_{3}(z+1) & \sim\left(\frac{z^{3}}{6}-\frac{z^{2}}{4}+\frac{1}{24}\right) \log (z+1)-\frac{11}{36} z^{3}+\frac{5}{24} z^{2}+\frac{z}{3}-\frac{13}{72} \\
& -\frac{z^{2}-z}{2} \zeta^{\prime}(0)+\frac{2 z-1}{2} \zeta^{\prime}(-1)-\frac{1}{2} \zeta^{\prime}(-2) \\
& +\frac{1}{12} \frac{1}{z+1}+\sum_{r=2}^{\infty}\left\{z^{2}-(6 r-11) z+\left(4 r^{2}-16 r+16\right)\right\} . \\
\log & G_{4}(z+1) \\
& \sim\left(\frac{z^{4}}{24}-\frac{z^{3}}{6}+\frac{z^{2}}{6}-\frac{19}{720}\right) \log (z+1) \\
& -\frac{4}{72} z^{4}+\frac{2}{9} z^{3}+\frac{z^{2}}{8}-\frac{11}{36} z+\frac{31}{144} \\
& -\frac{z^{3}-3 z^{2}+2 z}{6} \zeta^{\prime}(0)+\frac{3 z^{2}-6 z+2}{6} \zeta^{\prime}(-1)-\frac{z-1}{2} \zeta^{\prime}(-2)+\frac{1}{6} \zeta^{\prime}(-3) \\
& -\frac{1}{12} \frac{1}{z+1}+\frac{1}{720} \frac{1}{(z+1)^{3}}\left(6 z^{2}+\frac{13}{2} z+\frac{5}{2}\right) \\
& +\sum_{r=3}^{\infty} \frac{B_{2 r}}{[2 r]_{5}} \frac{1}{(z+1)^{2 r-1}}\left\{z^{3}-(12 r-27) z^{2}+\left(20 r^{2}-94 r+111\right) z\right. \\
& \left.-\left(8 r^{3}-56 r^{2}+134 r-109\right)\right\} .
\end{aligned}
$$


6. The Weierstrass product representation of the multiple gamma function. In this section, we derive the infinite product representation of the multiple gamma function more explicitly than (4). First, we observe the following proposition.

Proposition 4

$$
\exp \left(\zeta^{\prime}(-j)\right)=\exp \left(P_{j}(1)\right) \prod_{k=1}^{\infty}\left\{\left(1+\frac{1}{k}\right)^{\frac{B_{j+1}(k+1)}{j+1}} \exp \left(P_{j}(k+1)-P_{j}(k)\right)\right\}
$$

where

$$
\begin{aligned}
& P_{j}(x):=\sum_{r=0}^{j+1} \frac{B_{r}}{r !} \varphi_{j . r} x^{j-r+1}, \\
& \varphi_{j, r}:=\left.\left(\frac{d}{d t}\right)^{r}\left\{\frac{t^{j+1}}{j+1} \log t-\frac{t^{j+1}}{(j+1)^{2}}\right\}\right|_{t=1}
\end{aligned}
$$

and the infinite product converges absolutely.

This proposition is proved by using the Euler-MacLaurin summation formula and (10).

Similar calculation shows

Proposition 5

$$
\log G_{n}(z+1)=\sum_{j=0}^{n-1} G_{n, j}(z) K_{j}(z)
$$

where

$$
\begin{aligned}
K_{j}(z) & :=\frac{B_{j+1}(z+1)}{j+1} \log (z+1)-\zeta^{\prime}(-j)+P_{j}(z+1) \\
+\sum_{k=1}^{\infty} & {\left[P_{j}(z+k+1)-P_{j}(z+k)+\frac{B_{j+1}(z+k+1)}{j+1} \log \left(\frac{z+k+1}{z+k}\right)\right] . }
\end{aligned}
$$

Furthermore, the infinite sum of the last term is absolute convergent.

By using Proposition 4 and careful consideration on $K_{j}(z)$, we can see

$$
\begin{aligned}
K_{j}(z) & =Q_{j}(z)+\sum_{r=0}^{j-1}\left(\begin{array}{l}
j \\
r
\end{array}\right) z^{j-r} \zeta^{\prime}(-r)-\frac{z^{j+1}}{j+1} \gamma \\
+ & \sum_{k=1}^{\infty}\left\{-(z+k)^{k} \log \left(1+\frac{z}{k}\right)+\sum_{r=0}^{j}\left(\begin{array}{l}
j \\
r
\end{array}\right) z^{j-r} \sum_{l=1}^{j} \frac{(-1)^{l-1} z^{l}}{l} k^{r-l}\right\},
\end{aligned}
$$

where

$$
Q_{j}(z):=P_{j}(z+1)-\sum_{r=0}^{j}\left(\begin{array}{l}
j \\
r
\end{array}\right) z^{r} P_{j-r}(1)
$$




$$
+\frac{1}{j+1} \sum_{r=1}^{j+1}\left(\begin{array}{c}
j+1 \\
r
\end{array}\right) B_{j+1-r}(z) \sum_{l=1}^{r} \frac{(-1)^{l-1} z^{l}}{l} .
$$

The infinite sum in this formula converges absolutely.

Finally we can derive the following theorem:

TheOREM 6. For $n \in \mathbf{N}$, we have

$$
G_{n}(z+1)=\exp \left(F_{n}(z)\right) \prod_{k=1}^{\infty}\left\{\left(1+\frac{z}{k}\right)^{-\left(\begin{array}{c}
-k \\
n-1
\end{array}\right)} \exp \left(\Phi_{n}(z, k)\right)\right\}
$$

where

$$
\begin{aligned}
F_{n}(z) & :=\sum_{j=0}^{n-1} G_{n, j}(z) Q_{j}(z)+\sum_{r=0}^{n-2}\left[\frac{1}{r !}\left(\frac{\partial}{\partial u}\right)^{r}\left(\begin{array}{c}
z-u \\
n-1
\end{array}\right)\right]_{u=0}^{u=z} \times \zeta^{\prime}(-r) \\
& -\int_{0}^{z}\left(\begin{array}{c}
z-u \\
n-1
\end{array}\right) d u \times \gamma \\
\Phi_{n}(z, k) & :=\frac{1}{(n-1) !} \sum_{\mu=-1}^{n-2}\left\{\sum_{r=\mu+1}^{n-1} \frac{n-1}{r-\mu} z^{r-\mu}\right\}(-1)^{\mu+1} k^{\mu} .
\end{aligned}
$$

Examples of the Weierstrass product representation. By making use of Theorem 6, the Weierstrass product representation of the multiple gamma function is derived explicitly. We give some examples. In the case that $n=1$ and $n=2$, the results coincide with the Weierstrass representation of the gamma function and the $G$-function. In the case that $n=3$ and $n=4$, we obtain

$$
\begin{aligned}
G_{3}(z+1) & \exp \left\{-\frac{z^{3}}{4}+\frac{z^{2}}{8}+\frac{7}{24} z+\zeta^{\prime}(-1)-\frac{z(z-1)}{2} \zeta^{\prime}(0)-\left(\frac{z^{3}}{6}-\frac{z^{2}}{4} \gamma\right)\right\} \\
& \times \prod_{k=1}^{\infty}\left[\left(1+\frac{z}{k}\right)^{-\frac{k(k+1)}{2}} \exp \left\{\left(\frac{z^{3}}{6}-\frac{z^{2}}{4}\right) \frac{1}{k}-\left(\frac{z^{2}}{4}-\frac{z}{2}\right)+\frac{z}{2} k\right\}\right], \\
G_{4}(z+ & 1) \\
=\exp & \left\{\frac{61}{144} z^{4}+\frac{13}{18} z^{3}+\frac{19}{144} z^{2}-\frac{5}{24} z\right. \\
& \left.-\frac{z}{2} \zeta^{\prime}(-2)+\frac{z^{2}-2 z}{3} \zeta^{\prime}(-1)-\frac{z^{3}-3 z^{2}+2 z}{6} \zeta^{\prime}(0)-\frac{z^{4}-4 z^{3}+4 z^{2}}{24} \gamma\right\} \\
& \times \prod_{k=1}^{\infty}\left[( 1 + \frac { z } { k } ) ^ { \frac { k ( k + 1 ) ( k + 2 ) } { 6 } } \operatorname { e x p } \left\{\left(\frac{z^{4}}{24}-\frac{z^{3}}{6}+\frac{z^{2}}{6}\right) \frac{1}{k}\right.\right. \\
& \left.\left.-\left(\frac{z^{3}}{18}-\frac{z^{2}}{4}-\frac{z}{3}\right)+\left(\frac{z^{2}}{12}-\frac{z}{2}\right) k-\frac{z}{6} k^{2}\right\}\right] .
\end{aligned}
$$


Acknowledgements. The first author deeply thanks Professor S. Zakrzewski for his hospitality during the workshop at Banach Center.

The first author is partially supported by Grant-in-Aid for Scientific Research on Priority Area 231 "Infinite Analysis" and by Waseda University Grant for Special Research Project 95A-257.

\section{References}

[1] R. Askey, The q-Gamma and q-Beta functions, Appl. Anal. 8 (1978), pp. 125-141.

[2] E. W. Barnes, The theory of G-function, Quart. J. Math. 31 (1899), pp. 264-314.

[3] E. W. Barnes, Genesis of the double gamma function, Proc. London. Math. Soc. 31 (1900), pp. 358-381.

[4] E. W. Barnes, The theory of the double gamma function, Phil. Trans. Royal Soc. (A) 196 (1900), pp. 265-388.

[5] E. W. Barnes, On the theory of the multiple gamma functions, Trans. Cambridge Phil. Soc. 19 (1904), pp. 374-425.

[6] J. Dufresnoy et C. Pisot, Sur la relation fonctionnelle $f(x+1)-f(x)=\phi(x)$, Bull. Soc. Math. Belgique. 15 (1963), pp. 259-270.

[7] G. H. Hardy, On the expression of the double zeta-function and double gamma function in terms of elliptic functions, Trans. Cambridge. Phil. Soc. 20 (1905), pp. 395-427.

[8] G. H. Hardy, On double Fourier series and especially these which represent the double zeta-function and incommensurable parameters, Quart. J. Math. 37, (1906), pp. 53-79.

[9] F. H. Jackson, A generalization of the functions $\Gamma(n)$ and $x^{n}$, Proc. Roy. Soc. London. 74 (1904), pp. 64-72.

[10] F. H. Jackson, The basic gamma function and the elliptic functions, Proc. Roy. Soc. London. A 76 (1905), pp. 127-144.

[11] T. Koornwinder, Jacobi function as limit cases of q-ultraspherical polynomial, J. Math. Anal. and Appl 148 (1990), pp. 44-54.

[12] N. Kurokawa, Multiple sine functions and Selberg zeta functions, Proc. Japan. Acad. 67 A (1991), pp. 61-64.

[13] N. Kurokawa, Multiple zeta functions; an example, Adv. Studies. Pure. Math. 21 (1992), pp. 219-226.

[14] N. Kurokawa, Gamma factors and Plancherel measures, Proc. Japan. Acad. 68 A (1992), pp. 256-260.

[15] N. Kurokawa, On a q-analogues of multiple sine functions, RIMS. kokyuroku 843. (1992), pp. 1-10

[16] N. Kurokawa, Lectures delivered at Tokyo Institute of Technology, 1993.

[17] Yu. Manin, Lectures on Zeta Functions and Motives, Asterisque. 228 (1995), pp. 121-163.

[18] D. S. Moak, The q-analogue of Stirling Formula, Rocky Mountain J. Math,14 (1984),pp. 403-413.

[19] M. Nishizawa, On a q-analogue of the multiple gamma functions, to appear in Lett. Math. Phys. q-alg/9505086.

[20] T. Shintani, On a Kronecker limit formula for real quadratic fields, J. Fac. Sci. Univ. Tokyo Sect. 1A. Vol 24 (1977), pp 167-199.

[21] T. Shintani, A proof of Classical Kronecker limit formula, Tokyo J. Math. Vol.3 (1980), pp 191-199. 
[22] K. Ueno and M. Nishizawa, Quantum groups and zeta-functions in: J. Lukierski, Z.Popowicz and J.Sobczyk (eds.) "Quantum Groups : Formalism and Applications" Proceedings of the XXX-th Karpacz Winter School. pp. 115-126 Polish Scientific Publishers PWN. hep-th/9408143.

[23] K. Ueno and M. Nishizawa, in preparation.

[24] I. Vardi, Determinants of Laplacians and multiple gamma functions, SIAM. J. Math. Anal 19 (1988), pp. 493-507.

[25] M. F. Vignéras, L'équation fonctionnelle de la fonction zeta de Selberg de groupe modulaire $\operatorname{PSL}(2, Z)$, Asterisque. 61 (1979), pp. 235-249.

[26] A. Voros, Spectral functions, Special functions and the Selberg zeta functions, Comm. Math. Phys. 110 (1987), pp. 431-465.

[27] E. T. Whittaker and G. N. Watson, A Course of Modern Analysis, Fourth edition, Cambrige Univ. Press. 